\title{
Gradient Term Filtering for Stable Sound Propagation with Linearized Euler Equations
}

\author{
Xin Zhang ${ }^{1}$, Xiaoxian Chen ${ }^{2}$ and James Gill ${ }^{3}$ \\ Faculty of Engineering and the Environment \\ University of Southampton, Southampton, SO16 7QF, UK. \\ Xun Huang $^{4}$ \\ Department of Aeronautics and Astronautics \\ Peking University, Peking 10087, China
}

\begin{abstract}
A new, stable gradient term filtering (GTF) method is applied to time-domain linearised Euler equations (LEE) to compute sound propagation problems. The method employs a Laplace operator as a filter to obtain acoustic wave components. Through the filtering process, both the Kelvin-Helmholtz and the Rayleigh-Taylor instabilities can be removed from the solution process. Stability analysis confirms the stable behaviour of the solution in the presence of a sheared background mean flow, as against the conditional stability of LEE and gradient term suppression (GTS) methods. In accounting for vortical wave propagation, a curl operator can be conventionally utilised to obtain two-dimensional vortical wave components. Several benchmark test cases are studied to validate the proposed methods. Tests show that the proposed method can obtain stable solutions for acoustic wave propagation and is capable of modeling vortical interactions.
\end{abstract}

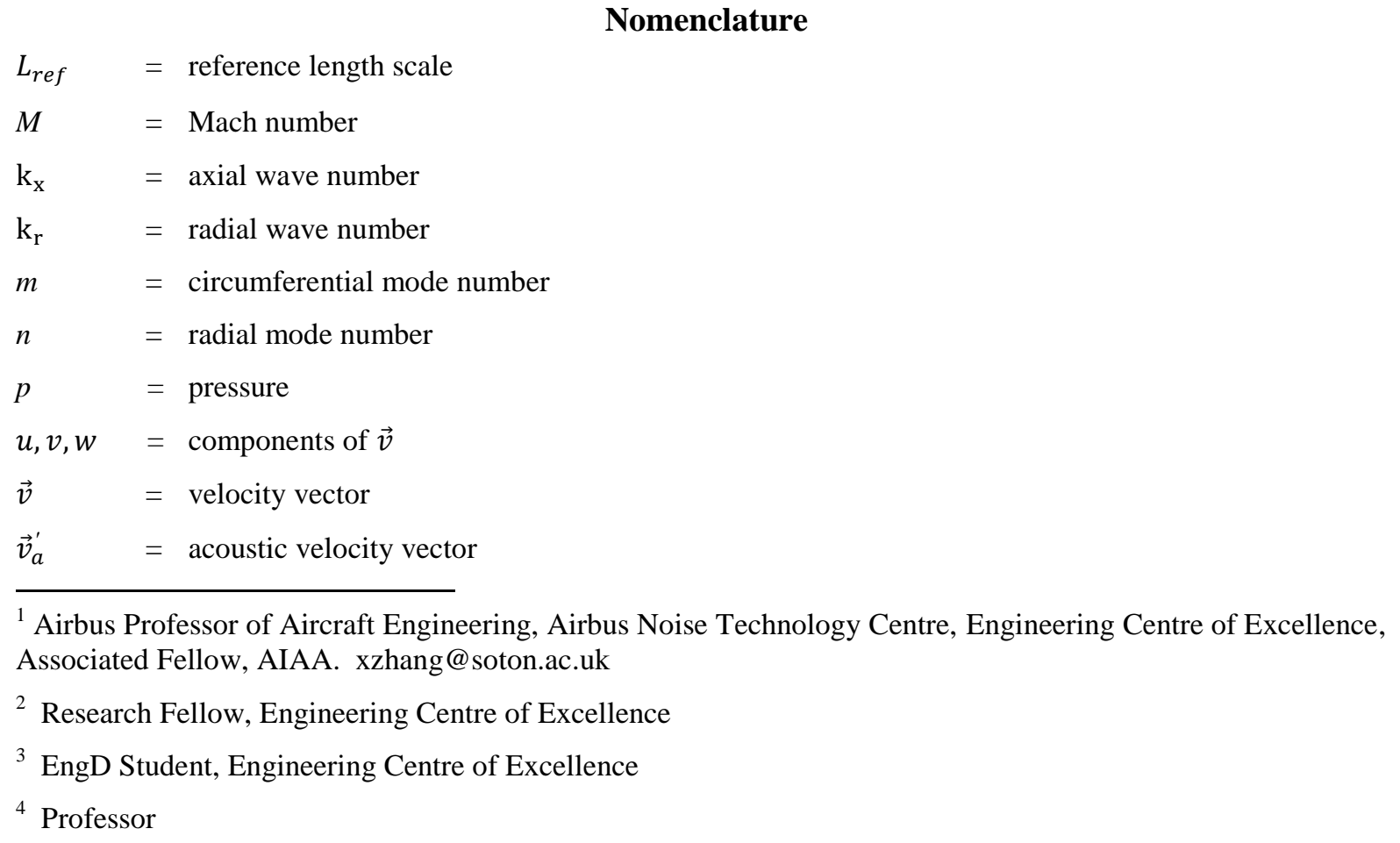

Nomenclature

$L_{\text {ref }} \quad=$ reference length scale

$M \quad=$ Mach number

$\mathrm{k}_{\mathrm{x}} \quad=$ axial wave number

$\mathrm{k}_{\mathrm{r}} \quad=$ radial wave number

$m=$ circumferential mode number

$n \quad=$ radial mode number

$p \quad=$ pressure

$u, v, w=$ components of $\vec{v}$

$\vec{v} \quad=$ velocity vector

$\vec{v}_{a}^{\prime} \quad=$ acoustic velocity vector

\footnotetext{
${ }^{1}$ Airbus Professor of Aircraft Engineering, Airbus Noise Technology Centre, Engineering Centre of Excellence, Associated Fellow, AIAA. xzhang@soton.ac.uk

${ }^{2}$ Research Fellow, Engineering Centre of Excellence

3 EngD Student, Engineering Centre of Excellence

4 Professor
} 


$$
\begin{aligned}
& \mathrm{x}, \mathrm{y}, \mathrm{z}=\text { non-dimensional axial, normal and span-wise components in Cartesian coordinates } \\
& \mathrm{x}, \mathrm{r}, \mathrm{\theta}=\text { non-dimensional axial, normal and azimuthal components in cylindrical polar coordinates }
\end{aligned}
$$

$$
\begin{array}{ll}
\text { Greek symbols } \\
\rho & =\text { density } \\
\delta & =\text { divergence }\left(=\nabla \cdot \vec{v}^{\prime}\right) \\
\phi & =\text { velocity potential } \\
\psi & =\text { vortical velocity potential } \\
\omega & =\text { frequency }(\mathrm{Hz}) \\
\nabla & =\text { gradient operator } \\
\nabla^{2} & =\text { Laplace operator } \\
\nabla \cdot \vec{v}^{\prime} & =\text { divergence operator } \\
\nabla \times \vec{v}^{\prime} & =\text { curl operator }
\end{array}
$$

Superscripts and subscripts

()$_{0}=$ background flow value in duct

()$^{\prime}=$ perturbation value
Abbreviations
GTF $=$ LEE employing gradient term filtering
GTS = LEE employing gradient term suppression
LDE-1 = first type of linearised divergence equation
LEE = linearised Euler equations
2.5D $=$ two-and-half dimensional

\section{Introduction}

$\mathrm{T}$ HE influence of noise on the environment and aircraft cabin attracts more and more attention when pushed by strict design requirements for future civil transport aircraft. There is an increasing demand to compute flow generated noise and sound propagation accurately, efficiently and robustly, for an aero-engine and for airframe noise applications specifically, and also for transport problems in general.

To predict the far-field noise radiation, an efficient approach is to use a version of the linearised Euler equations (LEE) as the governing equations for near-field sound propagation, ${ }^{1}$ and then to use a direct integral solution of noise radiation equations, e.g. Ffowcs Williams - Hawkings equation $(\mathrm{FW}-\mathrm{H})^{2}$ for the prediction of far-field directivity. However, in the time-domain, a solution based on LEE can suffer from instabilities due to background mean flow velocity or density gradients. The background mean aerodynamic flow often contains regions of local sheared flow (for airframe and engine applications) and regions of high temperature gradients (for engine applications). Of the various equations either currently being used in, or being developed by, industry and academia, the widely used LEE represent a class of acoustic approximation equations for sound propagation. ${ }^{3}$ They have advantages over various convected wave equations, in terms of physics, and also in terms of scalability for threedimensional computations. Therefore, they have a place in engineering computations. 
These linearised sound propagation equations, when used in the time-domain, can suffer from stability issues if the mean shear flow contains non-zero velocity or density gradients. ${ }^{4,5}$ Several methods have been developed to suppress these instabilities. A simplified version of LEE, ${ }^{4,5}$ using a gradient term suppression (GTS) method, was found to be able to suppress the Kelvin-Helmholtz instability by ignoring certain terms related to mean flow vorticity. However, such treatment may cause an imbalance in the energy transfer process and it also does not address the Rayleigh-Taylor instabilities that are likely to be present in background mean flows with density gradient. There is evidence that the gradient terms of the mean flow have a negligible effect on the sound pressure level (SPL) and the directivity pattern. ${ }^{6,7}$ Although GTS is often used in practice due to its simplicity and effectiveness ${ }^{4,5,7}$, it does suffer from a lack of physical justification. The GTS method also does not suppress Rayleigh-Taylor instability, since the pressure gradient term is not specifically treated.

Acoustic perturbation equations (APE) ${ }^{8}$ are another class of sound propagation equations that are derived from the Navier-Stokes equations using acoustic velocity components in the convective terms, and by filtering source terms with a Laplace operator. The high computing cost incurred by solving a Poisson equation is still a practical issue for time-domain CAA methods. The practical version (variant 4) of APE is also constrained by restrictive physical assumptions that require excessive user intervention, and is therefore difficult to use. A first type of linearised divergence equation (LDE-1) model $^{9}$ was developed for stable computations in situations with sheared background mean flows where time-domain LEE computations could be unstable. Furthermore, both the APE and the LDE-1 methods are only suitable for modelling the propagation of acoustic waves. They are unable to admit vortical disturbances, and therefore cannot be used in stochastic turbulence simulations, which are important for potential broadband noise studies.

In this work a new gradient term filtering (GTF) model is proposed to allow stable acoustic propagation computations in the time-domain. It is based on a fundamental theorem of vector calculus ${ }^{10}$ which states that any sufficiently smooth, rapidly decaying vector field can be resolved into the sum of an irrotational (curl-free) vector field and a solenoidal (divergence-free) vector field. From a physical point of view it is easy to understand that the acoustic wave (irrotational) is directly generated by flow expansion and contraction. In terms of formulation, the three linearised momentum equations can be written equivalently as one divergence equation plus one vorticity equation for two-dimensional (2D) flow or three vorticity equations for three-dimensional (3D) flow. For problems of acoustic wave propagation, the divergence equation is sufficient for the prediction of acoustic wave propagation as indicated by previous works. ${ }^{4,8,9}$ However, its full implementation is difficult, especially for acoustic spinning mode $(m>0)$ propagation in engine ducts, so that only a simplified model (LDE-1) is currently in use while a full version is being implemented and tested. The GTF method utilises an existing LEE solver, and solves a Poisson equation to obtain the acoustic velocity components to be used at the next time integration step. This process is reasonably straightforward to implement. Unlike the GTS and the LDE-1 methods, the solution obtained using the new GTF method is free from Rayleigh-Taylor instability. It can be applied to problems of acoustic propagation in engine exhaust flow and with jet - airframe interaction. For acoustic waves generated by vortical gust impingement on an airframe, a curl operator is used as a filter in order to obtain vortical velocity components of the particle velocity by solving the corresponding Poisson equations. Therefore, the GTF method has an advantage over the APE and the LDE-1 methods, because it is able to model the propagation of vortical disturbances.

In order to solve broadband sound propagation problems efficiently and robustly, the GTF method has also been developed for broadband acoustic noise computations. ${ }^{11}$ The model keeps the advantage of $2 \mathrm{D}$ form while solving a $3 \mathrm{D}$ problem by an assumption of a wave form in the azimuthal $(\theta)$ direction. This new formulation gives freedom in frequency by solving the acoustic variables such as acoustic pressure, velocity components in both real and imaginary spaces. All cut-on modes at different frequencies can be simulated in a single computation. Since modes between different frequencies are incoherent, the total computing cost for broadband noise is largely reduced. There is, however, a constraint on the new formulation, that if many individual frequencies are included in a single simulation. Estimation of SPL may require a long duration of computation to cover all periods of the acoustic modes. The same restriction also applies to the prediction of far-field radiation via an integral solution of FW-H equation. $^{2}$

In this paper, the new GTF method is described in detail and tested together with the GTS and the LDE-1 methods using a number of validation cases. These include benchmark test cases of sound propagation through a 2D turbulent flow, ${ }^{5,12}$ mode radiation from an unflanged engine duct featuring a jet with an infinitely thin shear layer (Munt's case), ${ }^{13,14}$ and sound induced by vortical gust impinging on an airfoil. ${ }^{21}$ For the Munt's case, the far-field directivity is computed through an integral solution of the FW-H equation, by reconstructing the 3D acoustic data on the entire integration surface from two-and-half dimensional (2.5D) computational results.

3 of 14

American Institute of Aeronautics and Astronautics 


\section{Formulation of the GTF method}

The GTF method utilises existing LEE while assuming that the momentum equation takes the form of divergence and vorticity equations implicitly. Using the vector form of the momentum equation, the LEE can be written below:

$$
\begin{aligned}
& \frac{\partial \vec{v}^{\prime}}{\partial t}+\left(\vec{v}^{\prime} \cdot \nabla\right) \vec{v}_{0}+\left(\vec{v}_{0} \cdot \nabla\right) \vec{v}^{\prime}+\frac{\nabla p^{\prime}}{\rho_{0}}-\frac{\nabla p_{0}}{\rho_{0}^{2}} \rho^{\prime}=\overrightarrow{0}, \\
& \frac{\partial p^{\prime}}{\partial t}+\vec{v}_{0} \cdot \nabla p^{\prime}+\vec{v}_{a}^{\prime} \cdot \nabla p_{0}+\gamma\left(p_{0} \delta^{\prime}+p^{\prime} \delta_{0}\right)=S_{p} \\
& \frac{\partial \rho^{\prime}}{\partial t}+\vec{v}_{0} \cdot \nabla \rho^{\prime}+\vec{v}_{a}^{\prime} \cdot \nabla \rho_{0}+\left(\rho_{0} \delta^{\prime}+\rho^{\prime} \delta_{0}\right)=S_{\rho},
\end{aligned}
$$

where $\gamma$ is the ratio of specific heats and source terms $S_{p}$ and $S_{\rho}$ indicate the source distribution. The velocity vector $\vec{v}$ has three components $u, v, w$ in either a Cartesian $(x, y, z)$ or a cylindrical polar $(x, r, \theta)$ coordinate system. $p$ and $\rho$ represent pressure and density respectively. Divergence $\delta$ and velocity potential $\phi$ and their relations with the velocity vector $\vec{v}$ in a cylindrical polar coordinate system are

$$
\delta^{\prime}=\nabla^{2} \phi=\nabla \cdot \vec{v}^{\prime}=\frac{\partial u^{\prime}}{\partial x}+\frac{\partial v^{\prime}}{\partial r}+\frac{\partial w^{\prime}}{r \partial \theta}+\frac{v^{\prime}}{r}
$$

where

$$
\nabla^{2} \phi=\frac{\partial^{2} \phi}{\partial x^{2}}+\frac{\partial^{2} \phi}{\partial r^{2}}+\frac{\partial^{2} \phi}{r^{2} \partial \theta^{2}}+\frac{\partial \phi}{r \partial r} .
$$

Before each time integration the acoustic velocity components are obtained from:

$$
u_{a}=\frac{\partial \phi}{\partial x}, \quad v_{a}=\frac{\partial \phi}{\partial r}, \quad w_{a}=\frac{\partial \phi}{r \partial \theta} .
$$

Vortical velocity components are easy to obtain in $2 \mathrm{D}$ flow in $(x, r)$ coordinates such that

$$
u_{v}=-\frac{\partial \psi_{\theta}}{\partial r}, \quad v_{v}=\frac{\partial \psi_{\theta}}{\partial x}, \quad w_{v}=0,
$$

where the vortical velocity potential $\psi$ is solved by another Laplace operator:

$$
\nabla^{2} \psi_{\theta}=\frac{\partial^{2} \psi_{\theta}}{\partial x^{2}}+\frac{\partial^{2} \psi_{\theta}}{\partial r^{2}}=\frac{\partial v^{\prime}}{\partial x}-\frac{\partial u^{\prime}}{\partial r}
$$

Eq. (8) is part of a vector equation, given by applying a curl operator $\nabla \times \vec{v}^{\prime}$ to the momentum Eq. (1).

$$
\nabla \times \vec{v}^{\prime}=\left(\frac{\partial v^{\prime}}{r \partial \theta}-\frac{\partial w^{\prime}}{\partial r}-\frac{w^{\prime}}{r}\right) \vec{\imath}_{x}+\left(\frac{\partial w^{\prime}}{\partial x}-\frac{\partial u^{\prime}}{r \partial \theta}\right) \vec{l}_{r}+\left(\frac{\partial v^{\prime}}{\partial x}-\frac{\partial u^{\prime}}{\partial r}\right) \vec{\imath}_{\theta},
$$

and we define

$$
\nabla \times \vec{v}^{\prime}=\nabla^{2} \psi_{x} \vec{\imath}_{x}+\nabla^{2} \psi_{r} \vec{\imath}_{r}+\nabla^{2} \psi_{\theta} \vec{\imath}_{\theta} .
$$

In a 3D flow, vortical velocity components are obtained from:

$$
u_{v}=-\frac{\partial \psi_{\theta}}{\partial r}-\frac{\partial \psi_{r}}{r \partial \theta}, \quad v_{v}=\frac{\partial \psi_{\theta}}{\partial x}+\frac{\partial \psi_{x}}{r \partial \theta}, \quad w_{v}=\frac{\partial \psi_{r}}{\partial x}-\frac{\partial \psi_{x}}{\partial r},
$$

In a 3D flow, use of the curl operator involves solving three Laplace operators and is time-consuming. Therefore, for the benchmark test cases involved, only $2 \mathrm{D}$ vortical waves $(m=0)$ are tested. The velocity potentials are solved using an iterative procedure with five iterations. ${ }^{9}$

The idea behind the GTF method is that a simple and stable model might be available for pure acoustic wave simulation, provided the velocity vector is constructed solely by acoustic velocity components. Numerical truncation errors or disturbances other than the acoustic components, due to the mean flow gradient effects, are filtered through a Laplace operator (i.e. Eq. (4)). As a whole, the GTF method provides a better physical justification than the GTS 
method. After each time integration step, the GTF method uses a divergence operator $\nabla \cdot \vec{v}^{\prime}$ to obtain acoustic velocity components. Then, the new acoustic velocity vector is used in LEE, implying that the term $\partial \vec{v}^{\prime} / \partial t$ tends towards $\partial \vec{v}_{a}^{\prime} / \partial t$. A 4th-order Runge-Kutta (R-K) time integration scheme ${ }^{9}$ is used in this work so that the new acoustic velocity vector is applied at each R-K stage. For acoustic waves induced by a vortical wave impinging on the airframe, an additional instability issue arises due to sheared flow, because the vortical waves are deformed by the sheared flow. GTF is as accurate as LEE when modeling vortical wave propagation.

For acoustic wave propagation alone, only the divergence equation is used, so that acoustic velocity components are used in Eqs. (1) to (3) and tests can be extended for acoustic spinning modes $(m>0)$. Furthermore, to include multi-frequency sources in a single computation for an engine duct spinning mode problem, ${ }^{11}$ each of Eqs. (1) to (3) can be separated into two equations in real and imaginary spaces by using a harmonic form $\left(\mathrm{e}^{-i m \theta}\right)$ of the solution in the azimuthal direction with an azimuthal wave number $m$ in a cylindrical polar coordinate system.

\section{Validations}

The proposed GTF method is validated against various benchmark test cases. These include the propagation of sound in a $2 \mathrm{D}$ turbulent shear flow, ${ }^{5,12,15}$ acoustic mode radiation out of an unflanged duct with a jet, ${ }^{13,14}$ and the noise produced by the interaction of a vortical gust with an airfoil leading edge. ${ }^{21}$ Three methods (GTS, GTF and LDE-1) will be tested and they have the potential to model the propagation of sound in sheared flow. Table 1 lists their features.

Table 1. Features of GTS, GTF and LDE-1 Methods.

\begin{tabular}{cr}
\hline GTS & LEE employing gradient term suppression (omitting terms associated \\
with $\partial u_{0} / \partial y$ and $\left.\partial v_{0} / \partial x\right)$ \\
GTF & LEE employing gradient term filtering (using divergence and curl operators) \\
LDE-1 & Simplified divergence equation assuming uniform mean density flow \\
\hline
\end{tabular}

In the computations, $\mathrm{a} 4^{\text {th }}$-order optimized spatial scheme was used for spatial derivatives and a low-dissipation, low-dispersion $4^{\text {th }}$-order Runge-Kutta scheme was used for time integration. A $10^{\text {th }}$-order explicit filter was applied to suppress any spurious waves developing in the calculations. Buffer-zones ${ }^{16}$ surrounding the computational domain maintained a reflectionless acoustic field. The simulations were performed on structured grids and the computational meshes used spatial resolutions of at least eight points-per-wavelength (PPW) in all directions. All variables are non-dimensionalised, unless otherwise mentioned, by the reference length of $L_{r e f}=1$ metre, the reference flow density $\rho_{r e f}$, and the reference flow sound speed $c_{r e f}$.

\section{A. Sound propagation through a 2D turbulent shear flow}

The first benchmark test case is the propagation of sound in a 2D turbulent shear flow. This is a challenging benchmark test since the mean turbulent shear layer features both velocity and density gradients containing both the Kelvin-Helmholtz and the Rayleigh-Taylor instabilities. To the authors' knowledge there are no time-domain LEE based methods that are able to successfully suppress the instability for this case unless special treatments, ${ }^{5,15}$ are adopted to remove the instability. In this study, a 2D computational domain was constructed within the range of $-140 \leq x \leq 140$ and $0 \leq y \leq 170$ in non-dimensionalised Cartesian coordinates, and cells were stretched near the shear layer $(0 \leq y \leq 5)$ along the $y$ direction, providing sufficient resolution for the mean flow profile. Apart from the lower boundary $(y=0)$, the entire computational domain was surrounded by outflow buffer zones to minimize spurious wave reflection into the computational domain. The total cell number was 147200 . With the reference values of $\rho_{\text {ref }}=p_{j} /\left(R T_{j}\right)$ and $c_{r e f}=\sqrt{\gamma R T_{j}}$ the mean non-dimensional velocity and density profile are shown in Error! Reference source not found. and they are:

$$
u_{0}(y)=u_{j}(y) e^{-\ln 2\left(\frac{y}{1.3}\right)^{2}}
$$




$$
\frac{1}{\rho_{0}(y)}=\rho_{\infty}\left\{-\frac{\gamma-1}{2 \gamma p_{j}}\left[u_{0}(y)-u_{j}\right] u_{0}(y)+\frac{u_{0}(y)}{\rho_{j} u_{j}}+\frac{u_{j}-u_{0}(y)}{\rho_{\infty} u_{j}}\right\},
$$

where flow parameters $u_{j}, \rho_{\infty}$ and $p_{j}$ are non-dimensional.

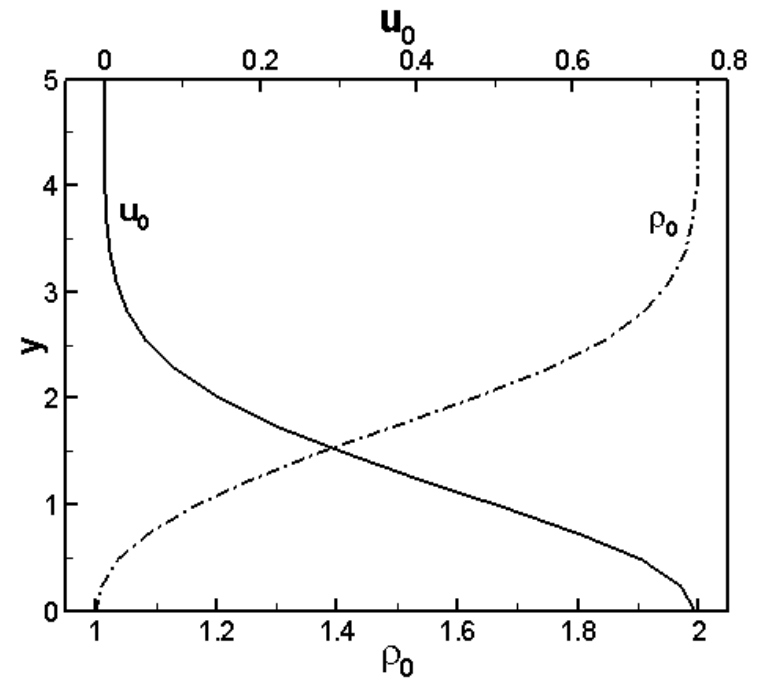

Figure 1. Mean $u$ velocity and density profiles.

Some flow parameters are listed in Table 2 . The case features a high temperature $\left(T_{j}=600 \mathrm{~K}\right)$ and high Mach number at the source.

Table 2: Mean Flow Parameters for Test Case A.

\begin{tabular}{ccccccc}
\hline$M\left(u_{0, y=0}\right)$ & $T_{j}$ & $T_{\infty}$ & $\omega$ & $p_{j} \cdot \rho_{\text {ref }} c_{\text {ref }}^{2}$ & $R$ & \\
& $(\mathrm{~K})$ & $(\mathrm{K})$ & $(\mathrm{Hz})$ & $\left(\mathrm{Kg} \cdot \mathrm{m}^{-1} \mathrm{~s}^{-2}\right)$ & $\left(\mathrm{m}^{2} \cdot \mathrm{K}^{-1} \mathrm{~s}^{-2}\right)$ & $\gamma$ \\
\hline 0.756 & 600 & 300 & 477.52 & 103330 & 287.0 & 1.4 \\
\hline
\end{tabular}

A source term $S_{p}(x, y)=0.001 e^{-\ln 2\left(0.04 x^{2}+0.32 y^{2}\right)} \cos (\omega t)$ is used on the right-hand side of Eq. (3) at the source location $x=0, y=0$. Figure 2 shows the non-dimensional acoustic pressures at the $60^{\text {th }}$ period. The computations based on the GTS and the GTF methods are stable, but the prediction of the LDE-1 method shows contaminated results within ten periods (see Fig. 2(c)). The two pressure fields predicted by GTS and GTF methods, shown in Figs. 2(a) and 2(b), are largely the same, featuring an upstream directed acoustic wave, and showing that the downstream unstable acoustic wave is removed. The removal of radial velocity gradients in GTS improves numerical stability while maintaining the accuracy of the final acoustic results.

It is noticed that the GTS and the LDE-1 methods are not developed to treat the appearance of the RayleighTaylor instability (i.e. not for sheared flow with density gradient) but GTS was stable within 60 periods of computation. At least it indicates that relative importance of two types of instabilities for practical computations. The Rayleigh-Taylor instability is less important than the Kelvin-Helmholtz instability. It also indicates that the LDE-1 method is sensitive to the Rayleigh-Taylor instability. 


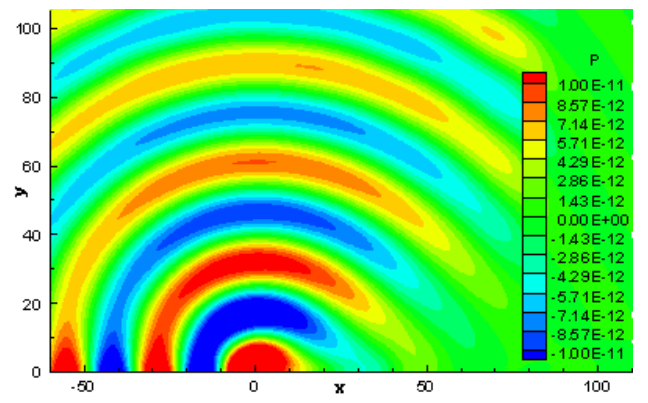

a) GTS.

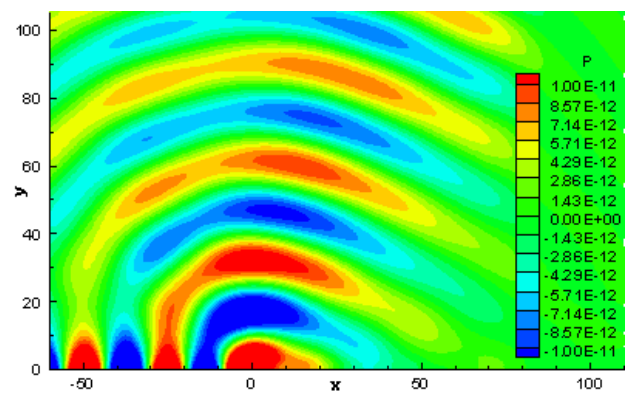

b) GTF.

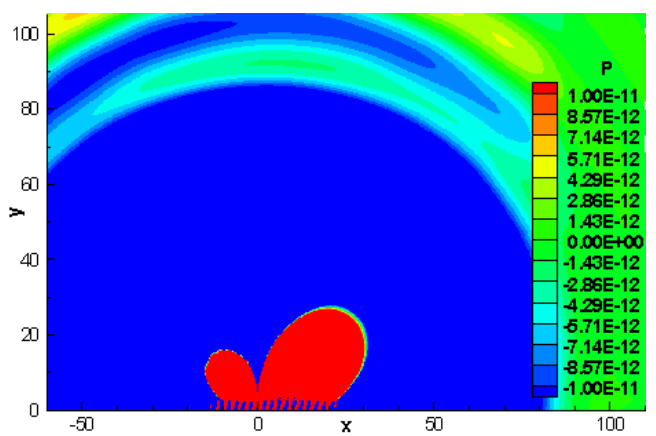

c) LDE-1 prediction at the $10^{\text {th }}$ period.

Figure 2. Acoustic pressure contours at the $60^{\text {th }}$ period.

\section{B. Sound radiation from an unflanged duct}

The second test case describes the mode radiation of an unflanged duct with a jet. ${ }^{13,14}$ Since an analytical farfield solution exists this test case is intended for developing a reliable far-field prediction with multi-frequency contents and used for comparison of the GTF method as well. In this case, the thickness of the shear layer is omitted. A detailed description of the computational setup can be found in Zhang and Chen. ${ }^{9}$ The computational domain contains a total of 32472 cells and the structure of the computational domain is shown in Figure 3. The problem is solved in cylindrical polar coordinates, where $(x, r)$ coordinates are shown in Figure 3, for an axi-symmetric mean flow.

The duct mean flow has a Mach number of 0.5 and there is no ambient mean flow over radius $r>1$. Since the main interest is of broadband predictions, the incident waves are constructed in the inflow buffer zone using a mode of $(m=6, n=1)$ at four individual frequencies $(500,1000,1500$ and $2000 \mathrm{~Hz}) . \quad m$ and $n$ are azimuthal and radial mode number. Results are sampled from the $50^{\text {th }}$ period onwards, and run for 250 periods of the highest modal frequency. Again, the three different methods are all numerically stable, yielding largely the same results. 


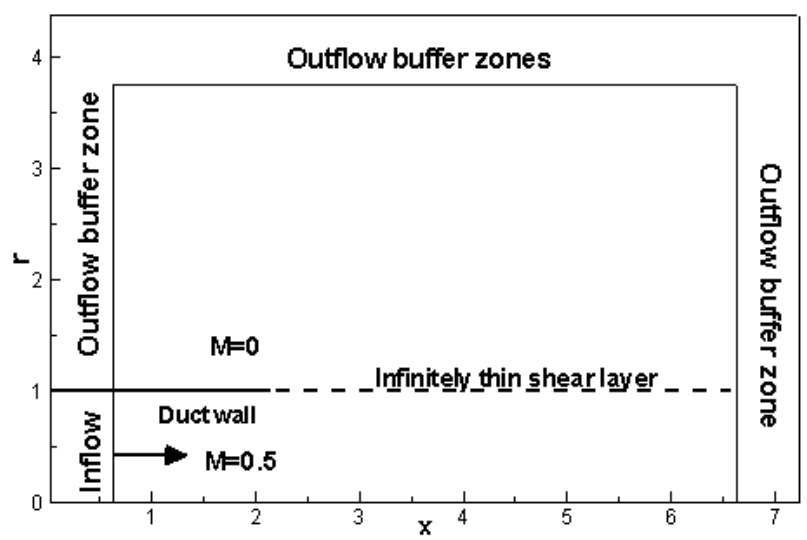

Figure 3. Computational domain and schematic of problem setup for unflanged duct case.

Figure 4 shows near-field SPL distributions obtained using the three methods (GTS, GTF and LDE-1). It can be seen that the overall sound pressure patterns are similar. In order to have a quantitative comparison, SPL distributions at $\mathrm{y}=1.5$ are shown in Figure 4d. the GTF method gives the highest peak value, which is $1.9 \mathrm{~dB}$ and 2.9 $\mathrm{dB}$ higher than the peak values of the GTS and the LDE-1 predictions respectively. Several factors may contribute to the discrepancies between the three methods, such as the amount of filtering, the type of governing equations and the iterative procedure. Additionally, the grid resolution around the duct opening will be verified in further study, in order to check that the discrepancies in near-field predictions are independent of the computational grid being used.

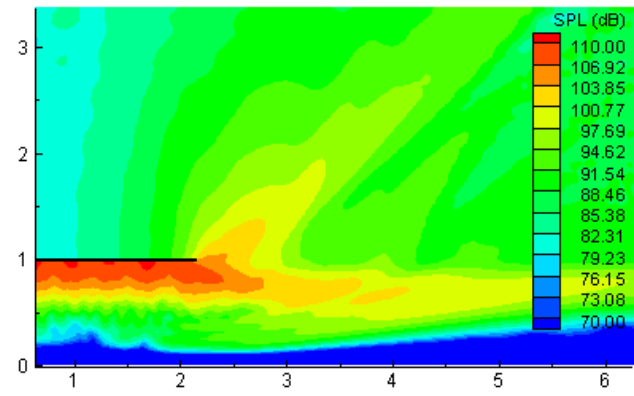

a) GTS.

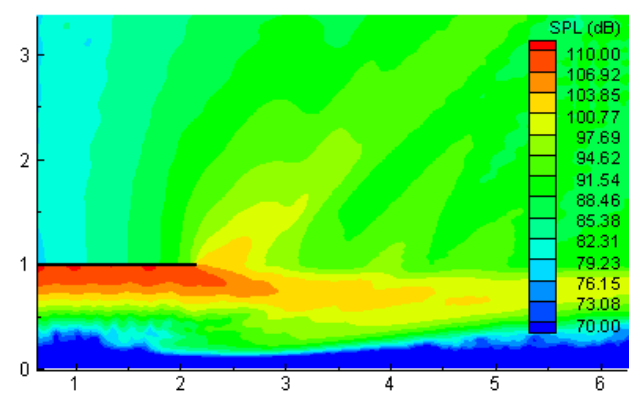

c) LDE-1.

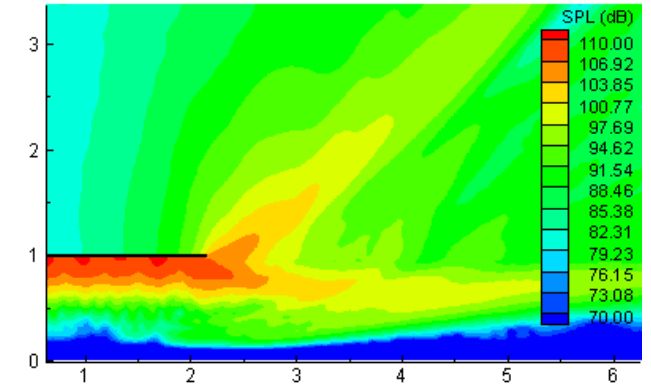

b) GTF.

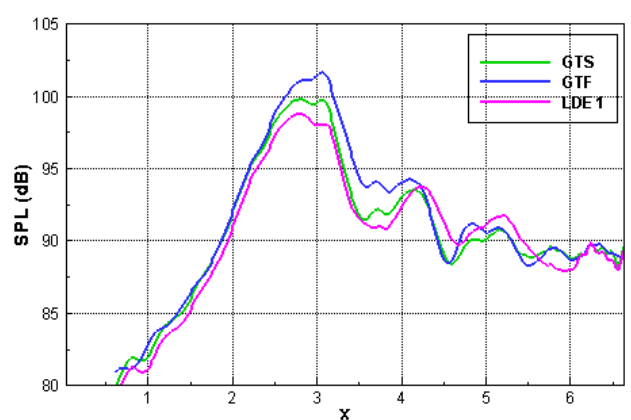

d) SPL comparison at a line of $y=1.5$.

Figure 4. Near-field sound radiation of an unflanged duct case.

The far-field directivity, containing multi-frequency content, is predicted through an integral solution of the FW$\mathrm{H}$ equation. ${ }^{2,17}$ Solution of the FW-H equation requires acoustic perturbation data on a 3D integration surface in the 
near-field. These data are presented in real values and can be reconstructed along the azimuthal $(\theta)$ direction from the 2.5D numerical solutions for an arbitrary variable $f(\theta, t)$,

$$
f(\theta, t)=f_{r}(0, t) \cos (m \theta)+f_{i}(0, t) \sin (m \theta)
$$

where $f_{r}(0, t)$ and $f_{i}(0, t)$ are the real and imaginary parts of variable $f$ at an angle $\theta=0$ respectively, and they are obtained from near-field predictions. The above formulation is different from the one previously used by Zhang et al. ${ }^{4}$ It is suitable for multi-frequencies while the earlier version is applied to a single frequency case using a time and azimuthal phase relation. To collect data for the integral solution, a grid resolution of $10 \mathrm{PPW}$ in the azimuthal direction and a resolution of 10 data samples per wave period for the highest modal frequency were found to be sufficient. With such a setup the FW-H solutions can be rapidly obtained within minutes on a personal computer (Intel processor, $2.2 \mathrm{GHz}$ and $2 \mathrm{~GB}$ memory) even for multi-frequency input.

Far-field directivity comparisons are plotted in Figure 5. For the GTF method, two peak radiation angles are observed at 50 and 56 degrees which are consistent with predictions using the other two methods and the analytical solution. The GTS method has a similar directivity pattern and SPL level as the GTF and the LDE-1 methods, which shows its effectiveness in this case. The peak observation (elevation) angle is determined by a cut-on ratio $\xi$ :

$$
\xi=\frac{\omega}{k_{r} \sqrt{1-M^{2}}}
$$

where $k_{r}$ is radial wave number. For a fixed azimuthal number the cut-on ratio reduces with increase of frequency. Therefore the main contribution from lowest frequency $(500 \mathrm{~Hz})$ will be at the higher observation angle (56 degrees) as the cut-on ratio is close to unity. The contribution from the highest frequency ( $2000 \mathrm{~Hz}$ ) will be mainly at the lower observation angle (50 degrees). At the lower peak angle, the differences in SPL between the CAA predictions (GTF, GTS and LDE-1 methods) and the analytical solution are $+0.3,-0.3$ and $-0.6 \mathrm{~dB}$ respectively. At the higher peak angle (56 degrees), the differences in SPL between the CAA predictions and the analytical solution are +1.7 , +0.1 and $-0.3 \mathrm{~dB}$ for the GTF, GTS and LDE- 1 methods respectively. In terms of accuracy, the three methods generally have similar performance. It is observed that the computation using the GTF method produces a nonsmooth directivity prediction, especially at lower angles ( $<45$ degrees). This test case will be further studied to confirm this result. Apart from the removal of the Rayleigh-Taylor instability, the GTF method is the same as the LDE-1 method in principle. There may be other factors affecting the outcome of a GTF computation, such as the use of less filtering to save computing time, and the grid resolution around duct opening.

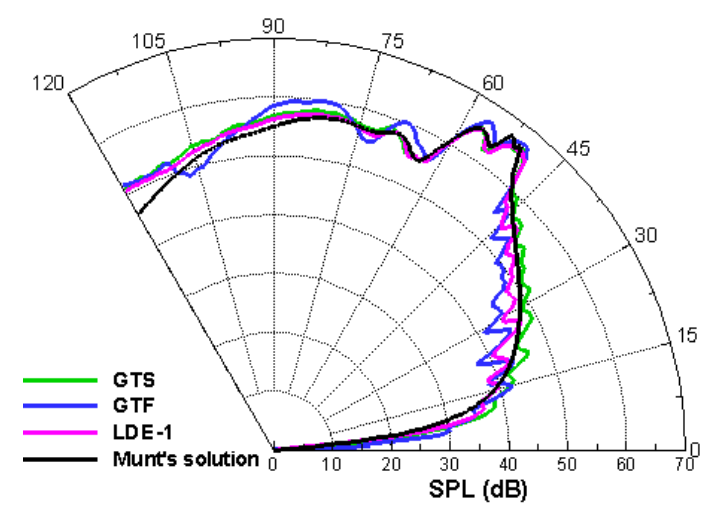

Figure 5. Comparison of far-field directivities.

Computing cost can be estimated for three methods. On a personal computer with an E4500 Intel processor (2.2 $\mathrm{GHz}$ clock speed) and 2.0 Gigabytes memory running on a 32-bit operating system, Table 3 summarises the computing time of three methods for an engine bypass duct case. The GTF method uses $23 \%$ more computing time than the GTS method, and is therefore the most expensive computing tool used in this test. The LDE-1 method uses 
less computing time since the Laplace operator is only applied at each time step rather than at each stage of the Runge-Kutta time marching scheme thus less iteration used in each time step.

Table 3: Comparison of Computing Costs

\begin{tabular}{cccc}
\hline Model & $\begin{array}{c}\text { Computing time } \\
\text { (seconds per grid } \\
\text { per time step) }\end{array}$ & $\begin{array}{c}\text { Total computing time } \\
\text { for 150 wave periods } \\
\text { (minutes) }\end{array}$ & $\begin{array}{c}\text { Ratio } \\
\text { (based on GTS) }\end{array}$ \\
\hline GTS & $1.01 \times 10-5$ & 230.1 & 1.00 \\
\hline GTF & $1.23 \times 10-5$ & 281.9 & 1.23 \\
\hline LDE-1 & $1.11 \times 10-5$ & 253.8 & 1.10 \\
\hline
\end{tabular}

\section{Sound induced by vortical gust impinging on an airfoil}

The case of a harmonic vortical wave interacting with the leading edge of an airfoil was also considered. This case is included to demonstrate that the GTF method is capable of propagating vortical disturbances in addition to acoustic disturbances. This represents an advantage over methods such as LDE-1. A single frequency sinusoidal wave was forced at the domain inflow buffer zone to have non-dimensional amplitude and wavelength of $0.01 \mathrm{M}$ and 0.125 chord lengths respectively. The wave was interacted with a NACA0002 airfoil in $M=0.3$ flow, where the nonuniform meanflow had been previously solved using an inviscid CFD computation. The domain used non-reflective boundary conditions on all faces to prevent spurious reflections interfering with the simulation. The noise was predicted for observers at a distance of 100 chord lengths from the airfoil using the FW-H prediction method previously discussed. This computational setup has been previously used in Gill et al. ${ }^{21}$

Figure 6 shows comparisons of the near-field acoustic pressure response between the LEE method and the proposed GTF method. The case could not be computed with the LDE-1 method, because it cannot propagate vortical disturbances. Figure 7 shows the directivity predictions made by the LEE and the GTF methods. The results in Figure 7 are also compared with an analytical flat plate solution, that was made using Amiet's theory, modified for $2 \mathrm{D}$ airfoils. ${ }^{22}$

A very similar directivity pattern is predicted by both methods. The positions of the directivity lobes, which are caused by interactions between the leading and trailing edge, are well predicted. Both CAA predictions show an over-prediction of the noise in comparison to the flat plate solution at upstream observer angles. This may be caused by the use of a NACA0002 airfoil as opposed to genuinely flat plate geometry. The GTF predictions differ from the LEE predictions at acute upstream angles, such that GTF predictions are approximately $2.5 \mathrm{~dB}$ louder than the LEE predictions. The reason for this is not clear, but may be associated with the velocity gradients in the leading edge stagnation region. Further testing would be required to ascertain whether the GTF or LEE methods provide a more accurate solution in this case. However, the agreement shown in Figure 7 is considered sufficient to demonstrate the ability of the GTF method to propagate vortical waves.

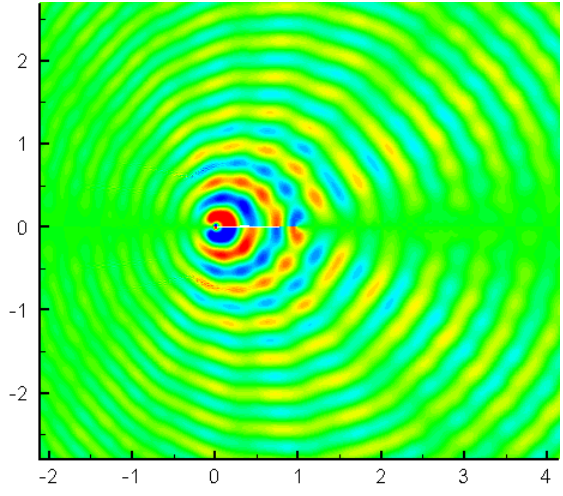

a) LEE.

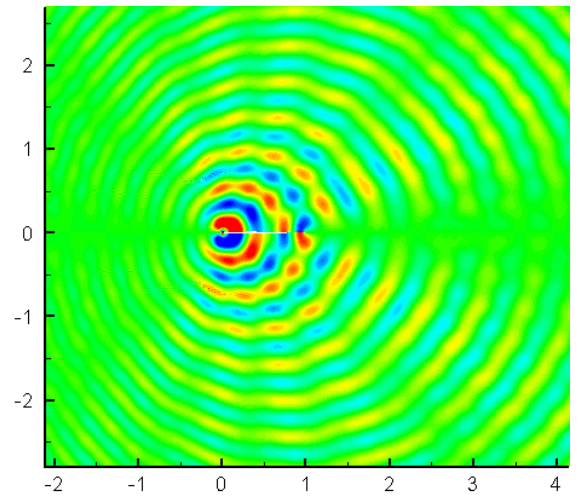

b) GTF.

Figure 6. Near-field acoustic pressure contours. 


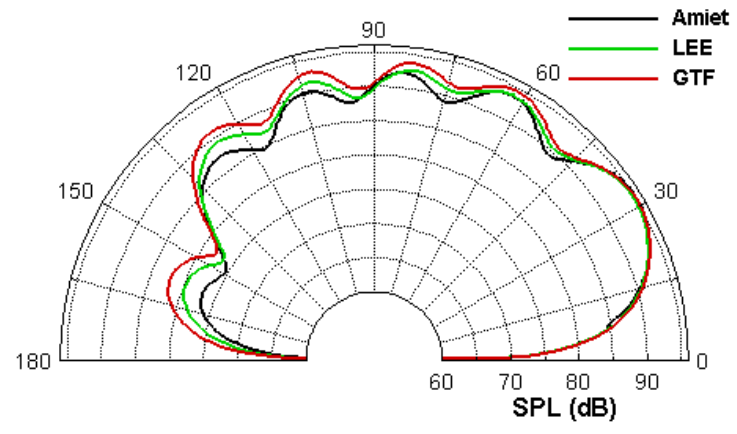

Figure 7. Far-field directivity.

\section{Stability Analysis}

The validations performed in the previous section have shown that the proposed GTF method produces stable solutions. The first validation case is a well-known unstable case for LEE due to the background mean sheared flow, and therefore a spatially unstable solution is developed along the axial $(x)$ direction. Stability analysis works exist for the LEE method in Cartesian coordinates ${ }^{5,18}$ based on the mean flow data. In this section, a stability analysis is performed in cylindrical polar coordinates for the LEE and the GTF methods. For comparison purposes, the stability analysis is performed for both the LEE and the GTF methods using a harmonic form of analysis.

To reduce the complexity of the system without losing generality, the following assumptions are made: $u_{0}=$ $u_{0}(r), \rho_{0}=\rho_{0}(r), v_{0}=0, w_{0}=0, p_{0}=$ constant, $p^{\prime}=\rho^{\prime}$. With emphasis on the mean shear flow, in this analysis the mean flow divergence influence on the model instability is omitted by setting $\delta_{0}=0$. With these assumptions the LEE can be derived in Eq. (14).

$$
\left\{\begin{array}{l}
\frac{\partial p^{\prime}}{\partial t}+u_{0} \frac{\partial p^{\prime}}{\partial x}+\gamma p_{0} \delta^{\prime}=0 \\
\frac{\partial u^{\prime}}{\partial t}+u_{0} \frac{\partial u^{\prime}}{\partial x}+\frac{\partial u_{0}}{\partial r} v^{\prime}+\frac{1}{\rho_{0}} \frac{\partial p^{\prime}}{\partial x}=0, \\
\frac{\partial v^{\prime}}{\partial t}+u_{0} \frac{\partial v^{\prime}}{\partial x}+\frac{1}{\rho_{0}} \frac{\partial p^{\prime}}{\partial r}=0, \\
\frac{\partial w^{\prime}}{\partial t}+u_{0} \frac{\partial w^{\prime}}{\partial x}+\frac{1}{\rho_{0}} \frac{\partial p^{\prime}}{r \partial \theta}=0 .
\end{array}\right.
$$

Substituting the harmonic form of the solutions,

$$
\left(p^{\prime}, u^{\prime}, v^{\prime}, w^{\prime}\right)^{T}=\left[A_{p}(r), A_{u}(r), A_{v}(r), A_{w}(r)\right]^{T} e^{i\left(\omega t-k_{x} x-m \theta\right)}
$$

into Eq. (14), a single equation for unknown spatial wave number $k_{x}$ can be derived in terms of pressure amplitude $A_{p}$,

$$
\left\{\frac{d^{2}}{d r^{2}}+\left[\frac{2 k_{x}}{D} \frac{d u_{0}}{d r}+\rho_{0} \frac{d}{d r}\left(\frac{1}{\rho_{0}}\right)+\alpha \frac{1}{r}\right] \frac{d}{d r}+\frac{\rho_{0} D^{2}}{\gamma p_{0}}-k_{x}^{2}-\frac{m^{2}}{r^{2}}\right\} A_{p}=0,
$$

where the parameter $D=\omega-k_{x} \mathrm{u}_{0}$. Eq. (15) is the same form as listed in references. ${ }^{19,20}$ It indicates that the instability comes from two sources, the velocity gradient term $\mathrm{du}_{0} / \mathrm{dr}$ and the density gradient term $\mathrm{d} \rho_{0} / \mathrm{dr}$ representing two instabilities: the Kelvin-Helmholtz instability and the Rayleigh-Taylor instability. As a special example for Cartesian coordinates $(\alpha=0)$ and 2D flow $(m=0)$, the above equation has a simple form.

$$
\left\{\frac{d^{2}}{d y^{2}}+\left[\frac{2 k_{x}}{D} \frac{d u_{0}}{d y}+\rho_{0} \frac{d}{d y}\left(\frac{1}{\rho_{0}}\right)\right] \frac{d}{d y}+\frac{\rho_{0} D^{2}}{\gamma p_{0}}-k_{x}^{2}\right\} A_{p}=0 .
$$

Eq. (16) is identical to the equations described in References ${ }^{5,18}$ where the harmonic form of the solutions takes a form of $e^{i\left(k_{x} x-\omega t\right)}$. For a given frequency $\omega$ a corresponding wave number $k_{x}$ can be obtained numerically by solving Eq. (16) using a shooting method with a classic Runge-Kutta fourth-order scheme. A numerical procedure is 
implemented to find the solution of $k_{x}$. If the wavenumber $k_{x}$ is a complex number $\left(k_{x}=k_{x r}+i k_{x i}, i=\sqrt{-1}\right)$ the LEE is spatially unstable. In this study, the negative sign of the $k_{x i}$ (spatial growing rate) shows the spatial instability.

In the benchmark case ${ }^{5,12,18}$, a periodic acoustic source is placed inside a flow shear layer. It is noted that the denominator $D$ in Eq. (16) has a non-zero complex value if the flow is unstable. Similar to Zheng et al, ${ }^{18}$ the acoustic wave is defined as an outgoing and decaying wave. In solving Eq. (16), the upper limit is set at $y=$ 25 which is sufficiently far away from $y=5$ where mean flow become uniform. Similar results are found within a frequency range from 0 to $565 \mathrm{~Hz}$ and for the excitation frequency of $477.52 \mathrm{~Hz}, k_{x}=0.41442-i 0.03773$.

It is also interesting to check if this instability could happen in cylindrical polar coordinates. Since additional terms appear in Eq. (15), which are proportional to the inverse of the radius to ensure similar wave behaviour at far field, the upper limit is extended to $r=60$. The maximum spatial growing rate is found at mode of $m=1$ and it is weak at other modes. Figure 8 shows the spatial growing rate and the real wave number. The value of the real wave number increases gradually with the frequency and there is a minimum value of the spatial growing rate around the frequency of $282.7 \mathrm{~Hz}$ showing the most unstable state. In other $m$ modes, no curve of the spatial rate can be found showing a stable state. Since the maximum spatial growing rate is found at mode $m=1$ it can be deduced that the instability most likely happens in lower $m$ modes.

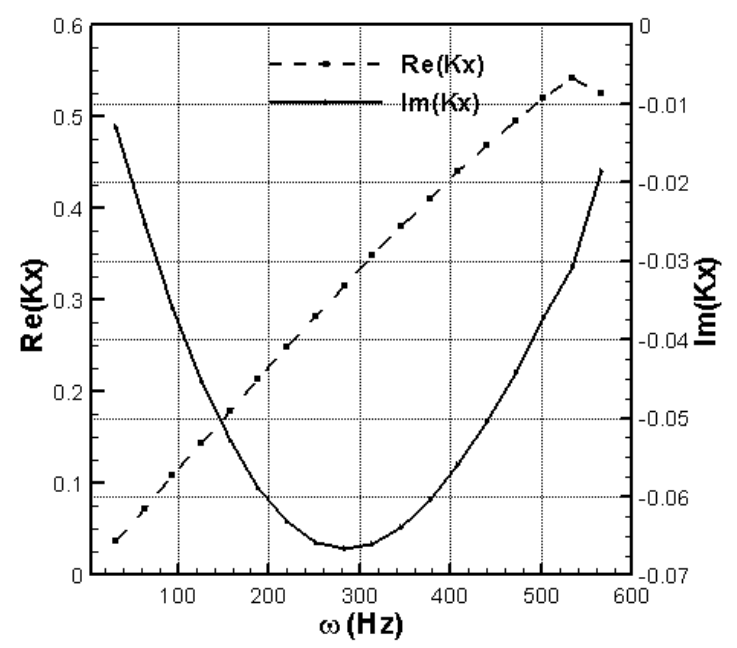

Figure 8. The characteristic wavenumber $k_{x}$ for a given excitation frequency $\omega$ at the mode of $m=1$ (LEE).

From the discussed assumptions, LEE contains only acoustic and vortical waves and it is proved that LEE is conditionally unstable.

The same stability analysis can be applied to the GTF method directly. Since only acoustic velocity components are used in the GTF method including the tendency term, the simplified GTF method can be written as follows using the divergence equation and the same assumptions:

$$
\left\{\begin{array}{l}
\frac{\partial p^{\prime}}{\partial t}+u_{0} \frac{\partial \mathrm{p}^{\prime}}{\partial x}+\gamma p_{0} \delta^{\prime}=0 \\
\frac{\partial \delta^{\prime}}{\partial t}+u_{0} \frac{\partial \delta^{\prime}}{\partial x}+2 \frac{\partial v^{\prime}}{\partial x} \frac{\partial u_{0}}{\partial r} \\
\quad+\frac{1}{\rho_{0}}\left(\frac{\partial^{2} p^{\prime}}{\partial x^{2}}+\frac{\partial^{2} p^{\prime}}{\partial r^{2}}+\frac{\partial^{2} p^{\prime}}{r^{2} \partial \theta^{2}}+\frac{1}{r} \frac{\partial p^{\prime}}{\partial r}\right)-\frac{1}{\rho_{0}^{2}} \frac{\partial \rho_{0}}{\partial r} \frac{\partial p^{\prime}}{\partial r}=0 \\
\delta^{\prime}=\frac{\partial^{2} \phi}{\partial x^{2}}+\frac{\partial^{2} \phi}{\partial r^{2}}+\frac{1}{r} \frac{\partial \phi}{\partial r}+\frac{\partial^{2} \phi}{r^{2} \partial \theta^{2}} \\
u^{\prime}=\frac{\partial \phi}{\partial x}, \quad v^{\prime}=\frac{\partial \phi}{\partial r}, \quad w^{\prime}=\frac{\partial \phi}{r \partial \theta}
\end{array}\right.
$$

Using harmonic wave assumption, Eq. (17) leads to two equations for the acoustic pressure amplitude $A_{p}$ and amplitude of the velocity potential $A_{\phi}$ : 


$$
\left\{\begin{array}{l}
{\left[\frac{d^{2}}{d r^{2}}+\left(\frac{\alpha}{r}-\frac{1}{\rho_{0}} \frac{d \rho_{0}}{d r}\right) \frac{d}{d r}+\frac{\rho_{0} D^{2}}{\gamma p_{0}}-k_{x}^{2}-\frac{m^{2}}{r^{2}}\right] A_{p}-i 2 \rho_{0} \frac{d u_{0}}{d r} k_{x} \frac{d A_{\phi}}{d r}=0} \\
\left(\frac{d^{2}}{d r^{2}}+\frac{1}{r} \frac{d}{d r}-k_{x}^{2}-\frac{m^{2}}{r^{2}}\right) A_{\phi}+i D A_{p} / \gamma p_{0}=0 .
\end{array}\right.
$$

The second-order ordinary differential equations can be cast into first-order equations. With predefined frequency $\omega$ and the wave number $m$, the solution of unknown $k_{x}$ can be solved numerically through Eq. (19) for $2 \mathrm{D}$ flow $(m=0)$ in Cartesian coordinates.

$$
\left\{\begin{array}{l}
\frac{d A_{\phi}}{d y}=B_{\phi} \\
\frac{d B_{\phi}}{d y}=k_{x}^{2} A_{\phi}-i D A_{p} / \gamma p_{0} \\
\frac{d A_{p}}{d y}=B_{p} \\
\frac{d B_{p}}{d y}=\frac{1}{\rho_{0}} \frac{d \rho_{0}}{d y} B_{p}-\left(\rho_{0} D^{2} / \gamma p_{0}-k_{x}^{2}\right) A_{p}+i 2 \rho_{0} \frac{d u_{0}}{d y} k_{x} B_{\phi} .
\end{array}\right.
$$

At lower boundary $(y=0)$ :

$$
B_{\phi}=0, \quad B_{p}=0 .
$$

At upper boundary: $\left.\frac{d u_{0}}{d y}\right|_{y \rightarrow \infty}=0,\left.\frac{d \rho_{0}}{d y}\right|_{y \rightarrow \infty}=0$,

$$
\left\{\begin{array}{l}
\frac{d^{2} A_{\phi}}{d y^{2}}-k_{x}^{2} A_{\phi}=-i k_{a} A_{p} / \gamma p_{0} \\
\frac{d^{2} A_{p}}{d y^{2}}-\left(k_{x}^{2}-\frac{\rho_{0}}{\gamma p_{0}} \omega^{2}\right) A_{p}=0 .
\end{array}\right.
$$

It can be established for the upper boundary assuming $k_{x r}>0$ to ensure wave decaying in $y$ direction):

$$
B_{p}=A_{p} \sqrt{k_{x}^{2}-\frac{\rho_{0}}{\gamma p_{0}} \omega^{2}}, \quad B_{\phi}=-k_{x} A_{\phi}-i \frac{\omega A_{p}}{\gamma p_{0}\left(\sqrt{k_{x}^{2}-\frac{\rho_{0}}{\gamma p_{0}} \omega^{2}}-k_{x}\right)} .
$$

In solving Eq. (19) no solution of spatial growing rate can be found indicating that the proposed GTF method is unconditionally stable.

In conclusion of the analysis, it is noted that both the vortical and the acoustic waves are contained in the LEE while only the acoustic wave is contained in GTF. It is also noted that in LEE the same equation (Eq. (14)) can be derived without the vortical wave components. From results of GTF it is known that instability exists for the acoustic wave but it cannot be excited by itself. Its excitation is from other disturbances such as the vortical wave as demonstrated in LEE. Extension of the stability analysis to the cylindrical polar coordinates gives the same conclusion.

\section{Conclusion}

A new and stable GTF method is proposed for acoustic propagation computations. Based on a fundamental theorem of vector calculus, the new model uses a divergence operator and a curl operator as filters to maintain acoustic and vortical components of the particle velocity by solving corresponding Poisson equations. The method is developed to overcome solution stability problems in solving some of the existing governing equations for acoustic propagation in time-domain. Stability analyses are performed in cylindrical polar coordinates for both the LEE and the GTF formulations using a benchmark case. Analyses show that the new GTF method is unconditionally stable while the original LEE method is conditionally unstable. 
Taking advantage of the stable characteristics of the new formulation, less filtering is needed, thus allowing savings in computation cost. The computational efficiency of the method is still comparable to that of the original LEE method in time-domain. In terms of computing cost, the GTF method is the most expensive one which uses $23 \%$ more time than the GTS method. However, in comparison to the LDE-1 method the GTF method is easier to implement.

Validation exercises are conducted against a number of benchmark cases which include the presence of background mean flow shear layers, and are known to generate unstable solutions using the LEE formulations. The results of the benchmark cases validate the efficiency, robustness, and accuracy of the new method. Results of the GTF method have also been compared with the GTS and the LDE-1 methods. This has confirmed the capability of the GTF method to compute sound propagation with background mean flows which contain velocity and density gradients. It also gives evidence that the GTS method has a rather small effect on the prediction of sound propagation in the near-field and noise radiation to the far-field. For challenging cases, with a background flow of velocity and density gradients, the GTF method can be used to obtain predictions of sound propagation.

\section{References}

${ }^{1}$ Zhang, X. "Aircraft noise and its nearfield propagation computations," Acta Mechanica Sinica, Vol. 28, No. 4, 2012, pp. 960-977.

${ }^{2}$ Ffowcs Williams, J.E., and Hawkings, D.L., "Sound generation by turbulence and surfaces in arbitrary motion," Philosophical Transactions of the Royal Society, London, Vol. 246, 1969, pp. 321-342.

${ }^{3}$ Rienstra, S.W., and Hirschberg, A., An introduction to acoustics, Eindhoven University of Technology, Netherlands, March 2014, pp.257-262, (http://www.win.tue.nl/ sjoerdr).

${ }^{4}$ Zhang, X., Chen, X.X., Morfey, C., and Nelson, P., "Computation of spinning modal radiation from an unflanged duct," AIAA Journal, Vol. 42, No. 9, 2004, pp. 1795-1801.

${ }^{5}$ Bailly, C., and Bogey, C., "Radiation and refraction of sound waves through a two-dimensional shear layer," $4^{\text {th }}-C A A$ workshop on benchmark problems, NASA/CP-2004-212954, 2004.

${ }^{6}$ Manera, J., Schiltz, B., Leneveu, R., Caro, S., and Jacqmot, J., "Kelvin-Helmholtz instabilities occurring at a nacelle exhaust," AIAA Paper 2008-2883, 2008.

${ }^{7}$ Leneveu, R., Schiltz, B., Laldjee, S., and Care, S., "Performance of a DGM scheme for LEE and applications to aircraft engine exhaust noise," AIAA Paper 2008-2884, 2008.

${ }^{8}$ Ewert, R., and Schröder, W., "Acoustic perturbation equations based on flow decomposition via source filtering," Journal of Computational Physics, Vol. 188, 2003, pp. 365-398.

${ }^{9}$ Zhang, X., and Chen, X.X., "Linearised Divergence Equations for Sound Propagation," AIAA Paper 2011-2930, 2011.

${ }^{10}$ Arfken, G.B. and Weber, H.J., Mathematical Methods for Physicists, International Edition, 6th edition, Academic Press: San Diego, 2005, pp. 95-101.

${ }^{11}$ Zhang X. and Chen, X.X., "Broadband wave propagation from an aero-engine duct," AIAA Journal, Vol. 52, No 1, pp. 43$51,2014$.

${ }^{12}$ Morris, P.J. and Agarwal, A., "Radiation and refraction of sound waves through a two-dimensional shear layer," 4 th $-C A A$ workshop on benchmark problems, Cleveland, Ohio, October 2003.

${ }^{13}$ Munt, R., "The interaction of sound with a subsonic jet issuing from a semi-infinite cylindrical pipe," Journal of Fluid Mechanics, Vol. 83, No. 4, 1977, pp. 609-640.

${ }^{14}$ Gabard, G., and Astley, R.J., "Theoretical model for sound radiation from annular jet pipes: far- and near-field solutions," Journal of Fluid Mechanics, Vol. 549, 2006, pp. 315-341.

${ }^{15} \mathrm{Li}$, X.D., Gao, J.H., Eschricht, D. and Thiele, F., "Numerical computation of the radiation and refraction of sound waves through a two-dimensional shear layer," $4^{\text {th }}-C A A$ workshop on benchmark problems, NASA/CP-2004-212954, 2004.

${ }^{16}$ Richards, S.K., Zhang, X., Chen, X.X. and Nelson, P.A., "Evaluation of non-reflecting boundary conditions for duct acoustic computation," Journal of Sound and Vibration, Vol. 270, No. 3, 2004, pp. 539-557.

${ }^{17}$ Farassat, F., Succi, G.P., "The prediction of helicopter discrete frequency noise," Vertica, Vol. 7, No. 4, 1983, pp. 309-320.

${ }^{18}$ Zheng, S., Miller, S.A.E. and Zhang, M., "Radiation and refraction of sound waves through a two-dimensional shear layer," $4^{\text {th }}-$ CAA workshop on benchmark problems, NASA/CP-2004-212954, 2004.

${ }^{19}$ Abid, M., Brachet, M., and Huerre, P., "Linear hydrodynamic instability of circular jets with thin shear layers," European Journal of Mechanics - B/Fluids, Vol. 12, No. 5, 1993, pp. 683-693.

${ }^{20}$ Michalke, A., "Survey on jet instability theory," Progress in Aerospace Sciences, Vol. 21, 1984, pp. 159-199.

${ }^{21}$ Gill, J., Zhang. X., and Joseph, P., "Symmetric airfoil geometry effects on leading edge noise," Journal of the acoustical Society of America, 134, 2013, pp. 2669-2680.

${ }^{22}$ Amiet, R., K., "Acoustic radiation from an airfoil in a turbulent stream," Journal of Sound and Vibration, Vol. 41, No. 4, 1975, pp. 407-420. 